\title{
A new look at dynamo cycle amplitudes
}

\author{
S.H. SAAR ${ }^{1}$ and A. BRANDENBURG ${ }^{2}$ \\ 1 Harvard-Smithsonian Center for Astrophysics, 60 Garden St., Cambridge, MA 02138, USA \\ 2 NORDITA, Blegdamsvej 17, DK-2100 Copenhagen Ø, Denmark
}

Received 2002 May 10; accepted 2002 July 15

\begin{abstract}
We explore the dependence of the amplitude of stellar dynamo cycle variability (as seen in the Mount Wilson Ca II HK timeseries data) on other stellar parameters. We find that the fractional cycle amplitude $A_{\text {cyc }}$ (i.e. the ratio of the peakto-peak variation to the average) decreases somewhat with mean activity, increases with decreasing effective temperature, but is not correlated with inverse Rossby number $R o^{-1}$. We find that $A_{\text {cyc }}$ increases with the ratio of cycle and rotational frequencies $\omega_{\text {cyc }} / \Omega$ along two, nearly parallel branches.
\end{abstract}

Key words: stars: magnetic fields - stars: activity - stars: chromospheres

\section{Introduction}

In a recent series of papers (Brandenburg, Saar, \& Turpin 1998; Saar \& Brandenburg 1999 [=SB], 2001), we have been exploring relationships between magnetic cycle periods $P_{\text {cyc }}$, rotation periods $P_{\text {rot }}$, and other stellar properties. By combining $P_{\text {cyc }}$ obtained from observations of Ca II emission (e.g., Baliunas et al. 1995 [=Bea95]), photometry, and $P_{\text {rot }}$ variation (e.g., Lanza \& Rodono 1999), we found evidence for trends between cycle $\left(\omega_{\text {cyc }}\right)$ and rotational $(\Omega)$ frequencies (Saar \& Brandenburg 2001), and between $\omega_{\text {cyc }} / \Omega$ and both $R_{\mathrm{HK}}^{\prime}$ and $R o^{-1}$ (Brandenburg et al. 1998; SB). Here, $R o^{-1}$ is the inverse Rossby number and $R_{\mathrm{HK}}^{\prime}$ is the Ca II HK flux, corrected for photospheric contributions and normalized by the bolometric flux (see Noyes et al. 1984).

Another important, but less studied observational property of stellar dynamos is the cycle amplitudes (i.e., the mean amplitude of the cyclic variability as seen in some activity diagnostic). There is a relationship between cycle amplitude and period seen in the Sun, where shorter cycles tend to be followed by stronger ones (e.g., Hathaway, Wilson \& Reichmann 1994), but there has been little work along these lines in stars. Focusing on inactive stars, Soon et al. (1994) suggested that the fractional peak-to-peak cycle amplitude seen in chromospheric Ca II HK emission,

$A_{\text {cyc }}=\Delta R_{\mathrm{HK}}^{\prime} /\left\langle R_{\mathrm{HK}}^{\prime}\right\rangle$,

decreased linearly with $\log \left(\Omega / \omega_{\text {cyc }}\right)^{2}$. Studying a larger sample, Baliunas et al. (1996) found that among inactive stars, $A_{\text {cyc }} \propto\left(\omega_{\text {cyc }} / \Omega\right)^{0.9}$. Results for active stars were less clear.

Correspondence to: saar@head-cfa.harvard.edu
Baliunas et al. (1996) analyzed all cycles detected by Bea95, independent of their quality. In our earlier work on cycle periods we found it very useful to begin by taking only a critically chosen sample of reliable, high grade cycles (e.g., Bradenburg et al. 1998, SB). In this paper, we therefore take a fresh look at cycle amplitudes, in light of the new results for $P_{\text {cyc }}$, using the same high quality cycle sample.

\section{Analysis}

We base our analysis on the Mount Wilson Ca II HK data (Bea95). Soon et al. (1994) gives cycle amplitudes for a number of inactive stars, and Baliunas et al. (1996) gives them for all stars with cycles in Bea95, but the $A_{\text {cyc }}$ values for common stars in these studies could not be reconciled. We have thus resorted, for this initial study, to "eye" estimates of the $S_{\mathrm{HK}}$ index amplitude $\Delta S_{\mathrm{HK}}$ (based on plots in Bea95) for stars not in Soon et al. (1994). These were converted into a $\Delta R_{\mathrm{HK}}^{\prime}$ following Noyes et al. (1984; see Table 1). As a test, we also made "eye" estimates of $\Delta S_{\mathrm{HK}}$ for stars in Soon et al. (1994) and found $\left\langle A_{\text {cyc }}(\right.$ eye $)-A_{\text {cyc }}($ Soon $\left.)\right\rangle=0.00 \pm 0.03$, indicating good agreement.

We studied stars chosen by SB for having well defined cycles, which in practice meant all cycles ranked "poor" in Bea95, and some graded "fair", were ignored. Stars were weighted by cycle grade, with an additional reduction applied if $A_{\text {cyc }}$ (eye) was unusually difficult to determine. Other stellar properties were also taken from the list compiled in SB. We estimated that the amplitude of the solar secondary $P_{\text {cyc }}$ (the Gleissberg cycle) at $\approx$ half of the primary (11 year) $A_{\text {cyc }}$. 
Table 1. Stellar Data

\begin{tabular}{|c|c|c|c|c|c|}
\hline HD \# & $B-V$ & $\begin{array}{l}-\log \\
R_{\mathrm{HK}}^{\prime}\end{array}$ & $A_{\mathrm{cyc}}^{*}$ & $\begin{array}{c}P_{\text {rot }} \\
\text { [d] }\end{array}$ & $\begin{array}{l}P_{\mathrm{cyc}}^{*} \\
{[\mathrm{yr}]}\end{array}$ \\
\hline Sun & 0.66 & 4.901 & 0.22 & 26.09 & $10.0,84$ \\
\hline 1835 & 0.66 & 4.433 & 0.15 & 7.78 & 7.78 \\
\hline 3651 & 0.85 & 4.991 & 0.36 & 44.0 & 14.6 \\
\hline 4628 & 0.88 & 4.852 & 0.38 & 38.5 & 8.60 \\
\hline 10476 & 0.84 & 4.912 & 0.38 & 35.2 & 9.60 \\
\hline 16160 & 0.98 & 4.958 & 0.32 & 48.0 & 13.2 \\
\hline 18256 & 0.43 & 4.722 & 0.16 & 3.0 & 6.8 \\
\hline 20630 & 0.68 & 4.420 & 0.14 & 9.24 & 5.6 \\
\hline 26913 & 0.70 & 4.391 & 0.13 & 7.15 & 7.6 \\
\hline 26965 & 0.82 & 4.872 & 0.38 & 43.0 & 10.1 \\
\hline 32147 & 1.06 & 4.948 & 0.42 & 48.0 & 11.1 \\
\hline 76151 & 0.67 & 4.659 & 0.16 & 15. & 2.52 \\
\hline 78366 & 0.76 & 4.608 & $0.27,0.19$ & 9.67 & $12.2,5.9$ \\
\hline 100180 & 0.57 & 4.922 & $0.17,0.07$ & 14. & $12.9,3.6$ \\
\hline 103095 & 0.75 & 4.896 & 0.27 & 31.0 & 7.30 \\
\hline 114710 & 0.57 & 4.475 & $0.21,0.12$ & 12.35 & $16.6,9.6$ \\
\hline 115404 & 0.93 & 4.480 & 0.16 & 18.46 & 12.4 \\
\hline 136202 & 0.54 & 5.088 & 0.21 & 16. & 23. \\
\hline 149661 & 0.82 & 4.583 & $0.35,0.15$ & 21.07 & $16.2,4.0$ \\
\hline 152391 & 0.61 & 4.448 & 0.28 & 11.43 & 10.9 \\
\hline 154417 & 0.57 & 4.533 & 0.17 & 7.78 & 7.4 \\
\hline 156026 & 1.16 & 4.662 & 0.37 & 21. & 21. \\
\hline 160346 & 0.96 & 4.795 & 0.44 & 36.4 & 7.00 \\
\hline 165341 & 0.86 & 4.548 & $0.54,0.12$ & 19.9 & $15.5,5.1$ \\
\hline 166620 & 0.87 & 4.955 & 0.30 & 42.4 & 15.8 \\
\hline 187691 & 0.55 & 5.026 & 0.11 & 10. & 5.4 \\
\hline 190007 & 1.17 & 4.692 & 0.30 & 28.9 & 13.7 \\
\hline 190406 & 0.60 & 4.797 & $0.34,0.15$ & 13.94 & $16.9,2.6$ \\
\hline 201091 & 1.18 & 4.764 & 0.32 & 35.37 & 7.30 \\
\hline 201092 & 1.37 & 4.891 & 0.21 & 37.84 & 11.7 \\
\hline 219834B & 0.91 & 4.944 & 0.29 & 43.0 & 10.0 \\
\hline
\end{tabular}

${ }^{*}$ data for a secondary cycle listed second where appropriate

\section{Results and discussion}

We first investigated how $\Delta R_{\mathrm{HK}}^{\prime}$ depends on $\left\langle R_{\mathrm{HK}}^{\prime}\right\rangle$ itself (Fig. 1). We find $\Delta R_{\mathrm{HK}}^{\prime} \propto\left\langle R_{\mathrm{HK}}^{\prime}\right\rangle^{0.77}(\sigma=0.17$ dex; fitting the primary $P_{\text {cyc }}$ only). This implies that $A_{\text {cyc }} \propto\left\langle R_{\mathrm{HK}}^{\prime}\right\rangle^{-0.23}$ - fractional cycle amplitudes decrease somewhat with increasing activity.

Some of the scatter about the fit can be explained by an additional dependence on $B-V$ color: $\Delta R_{\mathrm{HK}}^{\prime}$ is larger in $\mathrm{K}$ stars than $\mathrm{G}$ or $\mathrm{F}$ at fixed $R_{\mathrm{HK}}^{\prime}$. This is seen more clearly by plotting $A_{\text {cyc }}$ vs. $B-V$ color (Fig. 2), which shows an steady increase in $A_{\text {cyc }}$ from $\mathrm{F}$ through $\mathrm{G}$ stars, until a maximum is reached by the mid $\mathrm{K}$ stars $(B-V \sim 1.0)$. The averages by spectral type are $\left\langle A_{\text {cyc }}(\mathrm{F})\right\rangle=0.17,\left\langle A_{\text {cyc }}(\mathrm{G})\right\rangle=0.22$, and $\left\langle A_{\text {cyc }}(\mathrm{K})\right\rangle=0.35$. This behavior may be the result of a dependence of cycle amplitude on fractional convection zone depth, up to some limiting value in mid-K stars.

Some of the scatter in Fig. 1 is also intrinsic. If stellar cycles are like the Sun, activity will be restricted in latitude. Stars with different inclinations $i$ will then exhibit different apparent $A_{\text {cyc }}$ (Radick et al. 1998; Knaack et al. 2001). Analysis of the models in Knaack et al. (2001), however, indicates that $77 \%$ of the measured $A_{\text {cyc }}$ should lie within $\pm 15 \%$ of the mean, with only $23 \%$ (those with $i \leq 39^{\circ}$ ) will range from
$-15 \%$ to $-46 \%$ of $\left\langle A_{\text {cyc }}\right\rangle$. Furthermore, stars more active than the Sun are expected to have a wider latitude distribution of activity (e.g., Schrijver \& Title 2001), and thus should show less scatter due to varying $i$. Thus the effects of $i$ should be relatively small, and strongest stars with $R_{\mathrm{HK}}^{\prime} \lesssim R_{\mathrm{HK}}^{\prime}(\odot)$.

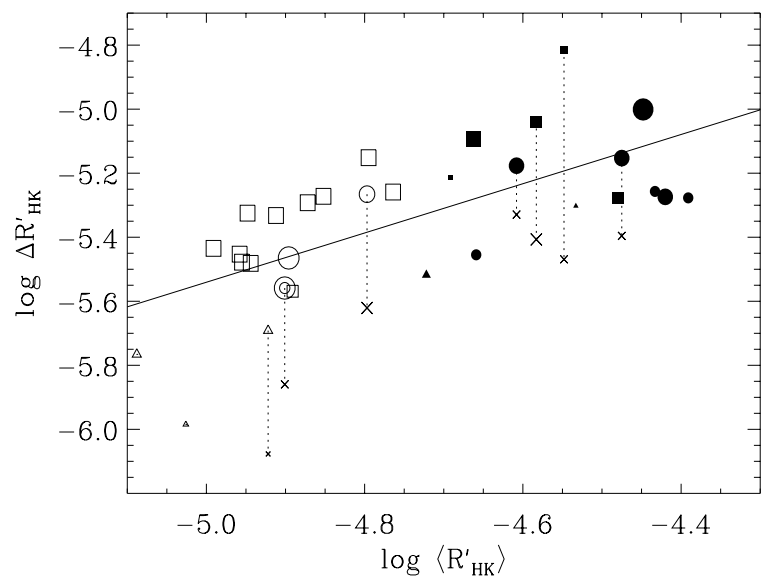

Fig. 1. $\Delta R_{\mathrm{HK}}^{\prime}$ vs. $\left\langle R_{\mathrm{HK}}^{\prime}\right\rangle$ for the stellar sample; symbols indicate $\mathrm{F}(\triangle), \mathrm{G}(\mathrm{o}$, the Sun's is doubled), and K (box) stars, filled symbols are more active stars, size $\propto P_{\text {cyc }}$ and $A_{\text {cyc }}$ "quality", dotted lines connect secondary $P_{\text {cyc }}(\mathrm{x})$ in some stars. We find $\Delta R_{\mathrm{HK}}^{\prime} \propto$ $\left\langle R_{\mathrm{HK}}^{\prime}\right\rangle^{0.77}$ (solid line), implying $A_{\mathrm{cyc}} \propto\left\langle R_{\mathrm{HK}}^{\prime}\right\rangle^{-0.23}$.

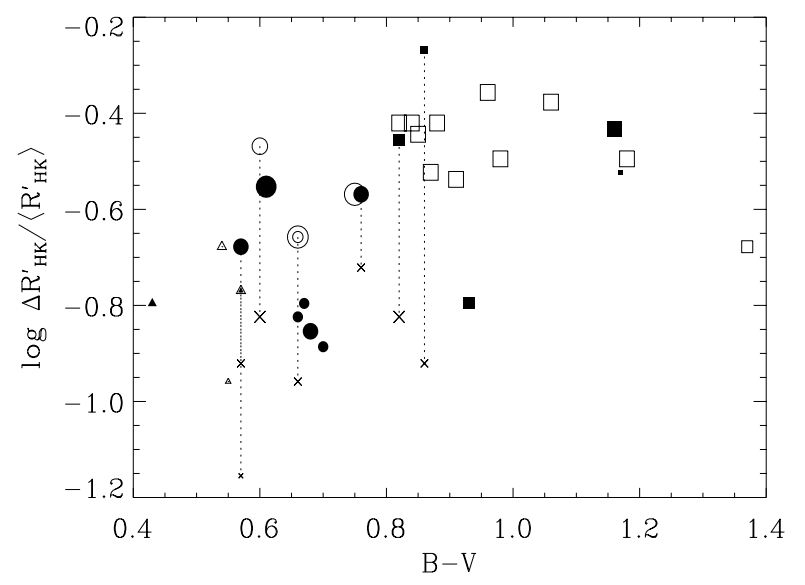

Fig. 2. $A_{\mathrm{cyc}}=\Delta R_{\mathrm{HK}}^{\prime} /\left\langle R_{\mathrm{HK}}^{\prime}\right\rangle$ vs. $B-V$ (symbols as in Fig. 1), showing an increasing $A_{\mathrm{cyc}}$ with decreasing effective temperature in $\mathrm{F}$ and $\mathrm{G}$ stars, reaching a maximum in mid-K stars.

In contrast to the dependence on $B-V, A_{\text {cyc }}$ shows little dependence on rotation, whether expressed as $\Omega$, or (see Fig. 3) as $R o^{-1}=\tau_{C} / P_{\text {rot }}$ (where $\tau_{C}$ is the convective turnover timescale; Noyes et al. 1984). Since $\left\langle R_{\mathrm{HK}}^{\prime}\right\rangle$ shows a clear dependence on $R o^{-1}$ (Noyes et al. 1984), this result implies $\Delta R_{\mathrm{HK}}^{\prime}$ has a similar dependence on rotation. Indeed, for $R o^{-1} \leq 1.5, \Delta R_{\mathrm{HK}}^{\prime} \propto R o^{-1}$ (above this $R o^{-1}$, there is evidence for cycle amplitude saturation). 
Since $A_{\text {cyc }}<1$, one might infer that only portion of $R_{\mathrm{HK}}^{\prime}$ derives from a cycling dynamo. This suggests that the noncycling (small-scale) component of the dynamo is prominent and thus (from Fig. 3) must have a similar dependence on rotation as the cycling component. However, this is at odds with the lack of any strong rotational dependence for $R_{\mathrm{HK}}^{\prime}$ in very inactive, "flat" activity stars where activity is likely fueled by a small-scale dynamo alone (Saar 1998). Thus, a more likely possibility is that there is significant temporal overlap in $R_{\mathrm{HK}}^{\prime}$ between cycles, reducing the apparent amplitude $A_{\text {cyc }}$. In this scenario, the cyclic dynamo dominates the rotational dependence of $R_{\mathrm{HK}}^{\prime}$, despite the apparently small $A_{\text {cyc. }}$. Enhanced cycle overlap with increasing $\left\langle R_{\mathrm{HK}}^{\prime}\right\rangle$ would also explain the decrease in $A_{\text {cyc }}$ with $\left\langle R_{\mathrm{HK}}^{\prime}\right\rangle$ (Fig. 1).

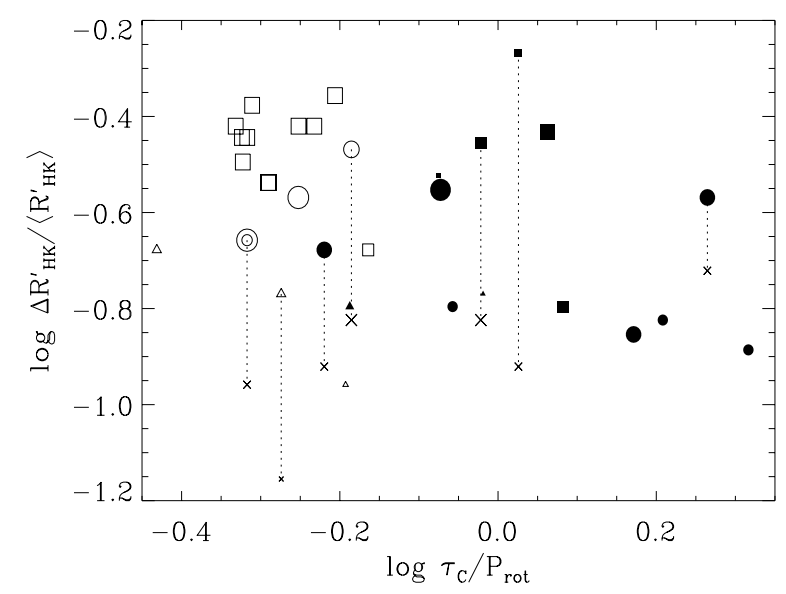

Fig. 3. $A_{\mathrm{cyc}}=\Delta R_{\mathrm{HK}}^{\prime} /\left\langle\Delta R_{\mathrm{HK}}^{\prime}\right\rangle$ vs. $R o^{-1}=\tau_{C} / P_{\text {rot }}$ (symbols as in Fig. 1), showing no clear relationship.

Study of the dependence of $A_{\text {cyc }}$ on $P_{\text {cyc }}$ with our dataset reveals a complex situation. Most inactive stars (defined as $\log R_{\mathrm{HK}}^{\prime} \leq-4.75$ ), combined with a few active ones, trace out a relation similar to that found by Baliunas et al. (1996), while most active ones show a similar, nearly parallel relation offset at higher $A_{\text {cyc. }}$. Specifically, 20 stars (14, or 70\% of them inactive) show $A_{\text {cyc }} \propto\left(\omega_{\text {cyc }} / \Omega\right)^{0.66}(\sigma=0.082$ dex), and 9 stars (7, or $78 \%$ of them active) exhibit $A_{\text {cyc }} \propto$ $\left(\omega_{\text {cyc }} / \Omega\right)^{0.85}(\sigma=0.081 \mathrm{dex})$. Only two lower quality detections cannot be assigned to one of these branches. This "branched" structure is similar to that seen between $\omega_{\text {cyc }} / \Omega$ and $R o^{-1}$ or $R_{\mathrm{HK}}^{\prime}$ by Brandenburg et al. (1998), SB, and Saar $\&$ Brandenburg (2001) (and first noted by Saar \& Baliunas 1992 and Soon et al. 1993). There are some differences in branch membership, though; for example, active G stars HD 1835,20630 , and 26913 lie on the active ("A") branch in SB, but the inactive ("I") branch here (Figs. 4, 5).

If we also consider secondary $P_{\text {cyc }}$, we find three lie on these branches, while four do not. Including the secondary $P_{\text {cyc }}$ in the branch fits causes little change: for the "I" branch, $A_{\text {cyc }} \propto\left(\omega_{\text {cyc }} / \Omega\right)^{0.68}(\sigma=0.082$ dex $)$ while for the "A" branch, $A_{\text {cyc }} \propto\left(\omega_{\text {cyc }} / \Omega\right)^{0.88}(\sigma=0.076$ dex $)$. Four of the stars show $A_{\text {cyc }}$ (primary) $/ A_{\text {cyc }}$ (secondary) $=2.26 \pm 0.18$, suggesting there may be some "preferred" amplitude ratios.

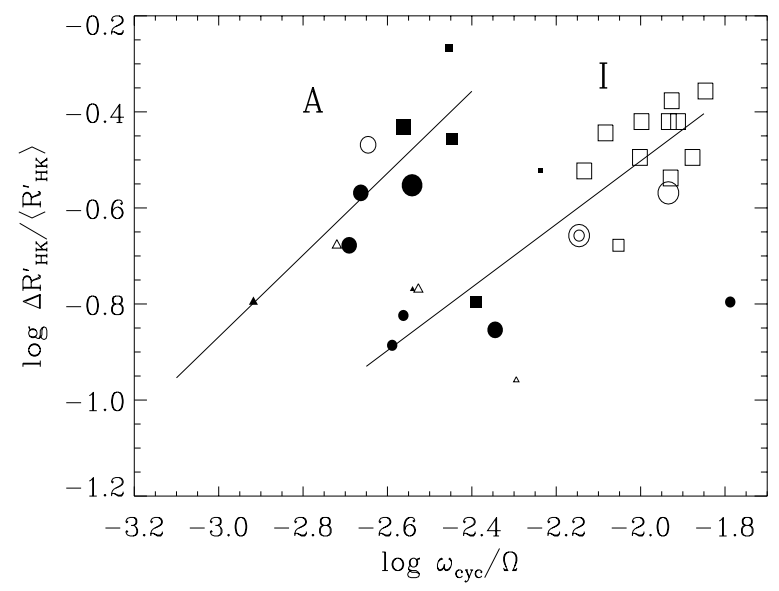

Fig. 4. $A_{\text {cyc }}=\Delta R_{\mathrm{HK}}^{\prime} /\left\langle\Delta R_{\mathrm{HK}}^{\prime}\right\rangle$ vs. $\omega_{\text {cyc }} / \Omega$ (symbols as in Fig. 1 ), showing an inactive (marked "I"; $\left.A_{\text {cyc }} \propto\left(\omega_{\text {cyc }} / \Omega\right)^{0.66}\right)$ and an active (marked "A"; $\left.A_{\text {cyc }} \propto\left(\omega_{\text {cyc }} / \Omega\right)^{0.85}\right)$ branch.

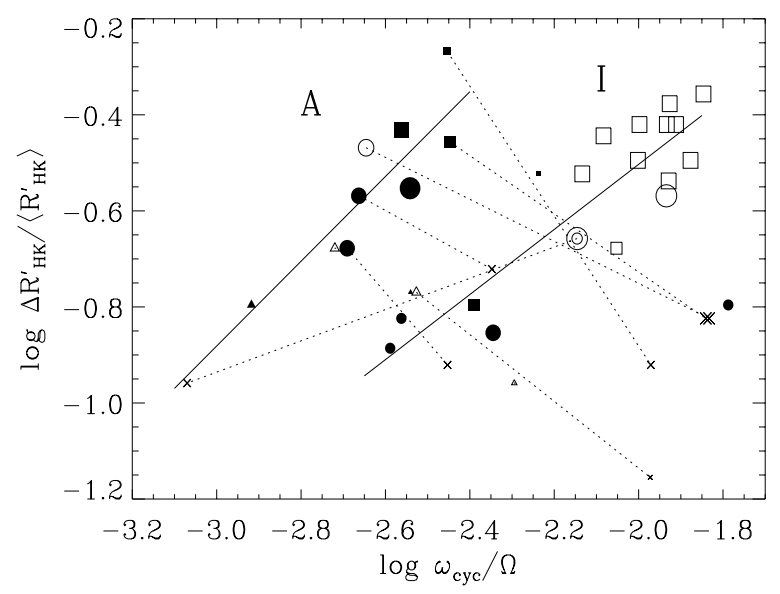

Fig. 5. Same as Fig. 4, but including secondary $P_{\text {cyc. }}$ An inactive (marked "I"; $\left.A_{\text {cyc }} \propto\left(\omega_{\text {cyc }} / \Omega\right)^{0.68}\right)$ and an active (marked "A"; $\left.A_{\text {cyc }} \propto\left(\omega_{\text {cyc }} / \Omega\right)^{0.88}\right)$ branch are indicated.

All four secondary cycles "unmatched" to a branch lie at low $A_{\text {cyc }}$ and high $\omega_{\text {cyc }} / \Omega$ and may indicate a third branch - more data is needed to confirm this.

Thus, it appears that $A_{\text {cyc }}$ and $P_{\text {cyc }}$ are related in stars, just as they are in the Sun. Study of stars indicates the relationship is multivalued, and depends on rotation.

The present analysis is limited due to the saturation of Ca II emission for $R o^{-1} \gtrsim 3$, which likely suppresses and obscures the visibility of cycles for more active stars. Cycle overlap is also a concern. A logical next step would be to investigate photometric cycle amplitudes, which in active stars are due to spots (rather than the plage/network seen in Ca II). Photometric cycle amplitudes saturate at considerably higher $R o^{-1}$ (Messina et al. 2001), permitting study of $A_{\text {cyc }}$ in much more active, faster rotating stars. Ideally, some normalized quantity like the fractional luminosity amplitude $\Delta L / L$ or the starspot filling factor $f_{S}$ (e.g., from TiO measurements) should be used. 
Acknowledgements. This research was supported by NSF grant AST-9731652, HST grant GO-8143, and NASA Origins grant NAG5-10630. We are indebted to the referee, K. Oláh for comments and suggestions, M. Dikpati for enlightening discussions, and to $\mathrm{S}$. Baliunas and R. Donahue for their initial advice and encouragement.

\section{References}

Baliunas, S.L., Donahue, R.A., Soon, W., et al.: 1995, ApJ 438, 269 (=Bea95)

Baliunas, S.L., Nesme-Ribes, E., Sokoloff, D., Soon, W.: 1996, ApJ 460,848

Brandenburg, A., Saar, S.H., Turpin, C.J.: 1998, ApJ 498, L51

Hathaway, D.H., Wilson, R.M., Reichmann, E.J.: 1994, SoPh 151, 177

Knaack, R., Fligge, M., Solanki, S.K., Unruh, Y.: 2001, A\&A 376, 1080
Lanza, A., Rodonò, M.: 1999, A\&A 349, 887

Messina, S., Rodonò, M., Guinan, E.F.: 2001, A\&A 366, 215

Noyes, R.W., Hartmann, L., Baliunas, S.L., Duncan, D.K., Vaughan, A.H.: 1984, ApJ 279, 763

Radick, R.R., Lockwood, G.W., Skiff, B.A., Baliunas, S.L.: 1998, ApJS 118, 239

Saar, S.H.: 1998, in: R.A. Donahue, J.A. Bookbinder (eds.), Cool Stars, Stellar Systems, and the Sun, ASP Conf. Ser. 154, 211

Saar, S.H., Baliunas, S.L.: 1992, in: K.L. Harvey (ed.), The Solar Cycle, ASP Conf. Ser. 27, 150

Saar, S.H., Brandenburg, A.: 1999, ApJ 524, 295 (=SB)

Saar, S.H., Brandenburg, A.: 2001, in: G. Mathys, S.K. Solanki, D. Wickramasinghe (eds.), Magnetic Fields Across the HR Diagram, ASP Conf. Ser. 248, 231

Schrijver, C.J., Title, A.M.: 2001, ApJ 551, 1099

Soon, W.H., Baliunas, S.L., Zhang, Q.: 1993, ApJ 414, L33

Soon, W.H., Baliunas, S.L., Zhang, Q.: 1994, SoPh 154, 385 